\title{
El ordenamiento territorial y su papel en la construcción de lugares saludables, caso: zona metropolitana de Toluca, México
}

\author{
Marcela Virginia Santana Juárez, \\ Elsa Mireya Rosales Estrada, Luis Ricardo Manzano \\ Solís, Giovanna Santana Castañeda y \\ Rebeca Angélica Serrano Barquín
}

\section{Resumen}

El objetivo de este trabajo es analizar el papel de la ordenación territorial en la construcción de lugares saludables, ante problemas ambientales y socioeconómicos que inciden negativamente en la salud de la población. Se considera un diagnóstico de las características socio-económicas, ambientales y de salud de la población de la Zona Metropolitana de Toluca (ZMT).

Metodología. Se trata de un estudio descriptivo, de carácter trans-seccional y el tipo de investigación es cuantitativo. Las fuentes de información son: imágenes de satélite, cartografía temática, Anuario Estadístico del Estado de México del Instituto Nacional de Estadística y Geografía (INEGI, 2011) y datos del Instituto de Salud del Estado de México (ISEM), entre otras fuentes.

Entre los resultados principales de la investigación destaca el hecho de que se trata de municipios con grado de marginación muy bajo y bajo; grado de rezago social muy bajo y medio; índice de desarrollo humano medio alto y alto; actividades económicas principalmente en servicios e industria; sin embargo se presentan problemas ambientales como erosión y falta de espacios verdes, así como tasas de mortalidad altas principalmente de tipo crónico degenerativo como la diabetes mellitus, enfermedades isquémicas del corazón, tumores malignos y cirrosis y otras enfermedades del hígado.

Ante estos problemas la ordenación territorial juega un papel importante en la generación de propuestas estratégicas focalizadas, en la creación de lugares saludables con un enfoque holístico y regional.

Palabras clave: Ordenación territorial. Lugares saludables. Salud humana. 


\section{Abstract}

The purpose of this paper is to analyze the role of spatial planning in building healthy places, to environmental and socioeconomic problems that negatively affect the health of the population. It is considered a diagnosis of the features socio-economic, environmental and population health of Toluca Metropolitan Area (ZMT).

Methodology. It is a descriptive study, cross-sectional and type of research is quantitative. The sources of information are: satellite images, thematic mapping, Statistical Yearbook of State of Mexico of National Institute of Statistics and Geography (INEGI, 2011) and data from the Health Institute of State of Mexico (ISEM), among other sources.

Among the main results of the research highlights the fact that it is municipalities with very low and low levels of marginalization; degree of social backwardness very low and medium; human development index is medium high and high; economic activities mainly in services and industry, however presents environmental problems such as soil erosion and lack of green spaces; as well as high mortality rates mainly of chronic degenerative as diabetes mellitus, ischemic heart diseases, malignant tumors and cirrhosis and other liver diseases.

Given these problems the spatial planning plays has important role in generating of focused strategic proposals for creating healthy places with a holistic and in this case regional.

Keywords: spatial planning / healthy places / human health /

Marcela Virginia Santana Juárez (mvsantana7@hotmail.com); Elsa Mireya Rosales Estrada (emre2000@hotmail.com); Luis Ricardo Manzano Solís (Irmanzanos@uaemex.mx); Giovanna Santana Castañeda (gioscas1@hotmail.com): Rebeca Angélica Serrano Barquín (rebecaserrano09@gmail.com). Facultad de Geografía, Universidad Autónoma del Estado de México. 


\section{INTRODUCCIÓN}

El acelerado y anárquico crecimiento urbano de muchas de las ciudades de América Latina y el impacto en la transformación del ambiente, enmarca indispensable la revalorización de la relación sociedad naturaleza, la cual incide en la calidad de vida de la población.

En México, según el INEGI, en el 2010 el 76\% de la población se ubica en ámbitos urbanos, con diferentes problemáticas ambientales, sociales, económicas, culturales y de salud que afectan la calidad de vida de la población y cada vez más las hacen inhabitables.

Ciudad saludable de acuerdo a Hancock Trevor y Duhl Leonard (1988) se define como: "Aquella ciudad que continuamente está mejorando y creando los ambientes físicos y sociales y expandiendo los recursos comunitarios que habilitan a la gente para apoyarse mutuamente en el desempeño de todas las funciones de la vida y para desarrollar su máximo potencial".

Dentro de este concepto es importante destacar que una ciudad saludable busca una "mejor calidad de vida" para su población. Debe de propiciar un ambiente físico-social que brinde bienestar a los habitantes de la misma.

México enfrenta problemáticas dentro del entorno urbano como: pobreza extrema, exclusiones sociales, falta de manejo de basura, deficiente provisión de agua potable, deterioro ambiental, escasez y precaria vivienda, falta de provisión y accesibilidad de servicios no solo de salud, sino además de los de saneamiento básico y energía eléctrica (Restrepo, Málaga, 2011). A partir de estas consideraciones, es importante señalar que el sustrato esencial y fundamental es el entorno geográfico, el cual ofrece alternativas susceptibles para incorporar usos urbanos y su óptima redistribución y utilización de los recursos naturales en pro de la sustentabilidad, así como para el desarrollo de ciudades saludables.

Una de las estrategias de la Organización Mundial de la Salud (1995), es la creación de ciudades saludables a partir de la promoción de la salud, con el apoyo de organismos de diferentes ámbitos de la ciudad: servicios públicos, empresarial, políticos y la ciudadanía, entre otros. Priorizando los determinantes de la salud, las personas que viven en la pobreza y las necesidades de los grupos vulnerables (ejemplo: envejecimiento sano). En que las ciudades deben de trabajar en los temas de desarrollo básicos de la planificación urbana saludable (plan de desarrollo de ciudad saludable) y la evaluación de los efectos sobre la salud. 
La Organización Panamericana de la Salud (2005) ha impulsado el programa de municipios, ciudades y comunidades saludables. A partir de la estrategia internacional de la promoción de la salud para lograr equidad, democracia y justicia social, ofreciendo un marco valioso para organizar la acción social y política con el fin de mejorar la salud y las condiciones de vida.

El crecimiento de una ciudad saludable debe partir de los conceptos de sitio (emplazamiento) y situación los cuales tienen relación con las características físico geográficas, naturales, socioeconómicas, culturales y de salud, directamente vinculadas al desarrollo sustentable, que atiende la adecuada distribución y redistribución de los usos urbanos del suelo. La planificación territorial ha sido uno de los instrumentos de mayor alcance utilizado en América Latina para intervenir sobre los territorios, especialmente a través de los planes de ordenamiento territorial (Massiris, 2011). Por lo que es fundamental la concertación de todos los actores que inciden en la ciudad, desde el individuo, la población, los gobiernos, las instituciones educativas, las ecológicas, las de desarrollo urbano, hasta las instituciones internacionales, para plantear alternativas de solución y construir las ciudades saludables.

Joaquín Farinós señala la importancia de la participación e involucración de la población en la definición de alternativas de futuro (estrategias territoriales 0 spatial visions), para lo cual es necesaria la creación - lenta pero sin pausa- de nuevos lazos socio-territoriales (Farinós, 2011).

En Río de Janeiro, en la Conferencia de Naciones Unidas para el Medio de 1992 se estableció la Agenda 21: un documento que analiza y propone políticas para alcanzar el desarrollo sustentable de las ciudades, contempla en la sección 1 las dimensiones sociales y económicas y el apartado sobre la protección y fomento de la salud humana. En la cual señala que la salud y el desarrollo tienen una relación directa. Tanto el desarrollo insuficiente que conduce a la pobreza como el desarrollo inadecuado que redunda en el consumo excesivo, combinados con el crecimiento de la población mundial, que pueden redundar en graves problemas de salud relacionados con el medio ambiente en los países desarrollados y en los países en desarrollo.

Una ciudad saludable es aquella que da una alta prioridad a la salud en todas sus actuaciones. Cualquier ciudad puede ser saludable si se compromete con la salud, tiene una estructura para trabajar por ella y comienza un proceso para conseguir más salud. La salud se define como el bienestar físico, psíquico, social y medioambiental de la gente que vive y trabaja en las ciudades. 
La salud es algo más que la ausencia de enfermedad o la actividad curativa de los servicios sanitarios: puede crearse si el entorno donde vivimos nos facilita estilos de vida más sanos.

Algunos indicadores de una ciudad saludable son: el \% de personas que hacen ejercicio regularmente; la proporción de médicos para cada 100,000 personas; el porcentaje de personas derechohabiente; tasas bajas en enfermedades crónico degenerativas; bajos indicadores en la presión arterial, colesterol y triglicéridos; calidad del aire que se respira alta; ciudades limpias, ciudades con proporciones altas de áreas verdes, sistema de transporte funcional; entre otros.

El analizar las condiciones de salud en la Zona Metropolitana de Toluca (ZMT), permitirá realizar un diagnóstico como insumo en la ordenación territorial para la construcción de una ciudad saludable. No se trata de transformar por completo una ciudad, sino de localizar lugares estratégicos para políticas y estrategias que tengan mayor impacto en la salud de la población.

En este trabajo se presentan avances del proyecto de investigación sobre ciudades saludables en México, a partir de una perspectiva geográfica. Caso ZMT. En específico sobre algunas características ambientales, socioeconómicas y de salud de la población, con un enfoque regional.

\section{MÉTODOS}

El estudio es de carácter descriptivo, cuantitativo, de carácter trans-seccional, se analizan las características sociodemográficas, ambientales y de salud, de la ZMT como insumos en la ordenación territorial para la construcción de lugares saludables.

\subsection{Universo de estudio}

La ZMT se localiza en el centro del Estado de México, de acuerdo al INEGI, CONAPO y SEDESOL (2005) se integra por catorce municipios que tienen su propias especificidades, estos son: Almoloya de Juárez, Calimaya, Chapultepec, Lerma, Metepec, Mexicaltzingo, Ocoyoacac, Otzolotepec, Rayón, San Antonio la Isla, San Mateo Atenco, Toluca, Xonacatlán y Zinacantepec (INEGI, 2010).

La ZMT se localiza a los 2300 msnm, en el altiplano mexicano, forma parte del eje neo volcánico ubicado a los $19^{\circ}$ grados de latitud norte. Está integrada por una serie de montañas, lomas y valles en los que predomina el clima templado subhúmedo y en las prtes altas el semifrío. 
La ZMT ocupa el quinto lugar en tamaño de población, después de la Zona Metropolitana del Valle de México, la Zona Metropolitana de Monterrey, la Zona Metropolitana de Guadalajara y la Zona Metropolitana de Puebla. Con base en el XIII censo de población y vivienda 2010, la ZMT cuenta con 1, 846,116 habitantes, que indica el $12.16 \%$ de la población del Estado de México.

\subsection{Fuentes e información}

Las fuentes de información utilizadas son: imágenes de satélite, cartografía temática, Anuario Estadístico del Estado de México del Instituto Nacional de Estadística y Geografía (INEGI, 2011) y datos del Instituto de Salud del Estado de México (ISEM), entre otras.

\section{RESULTADOS}

\subsection{Distribución de la población}

La ZMT con una extensión de 2,038.35 km² y una densidad de $907 \mathrm{hab} /$ $\mathrm{km}^{2}$. Destacan entre los municipios con mayor densidad de población: Metepec con 3,171.83 hab/km²; San Mateo Atenco con 2,650.80 hab/km²; y Toluca con 1,811.71 hab/ $/ \mathrm{km}^{2}$. La distribución de la población según el tamaño de localidad se presenta principalmente urbana (población que vive en localidades con más de 15,000 habitantes), con el 55.34\%; mixta el $29.36 \%$ (población que vive en localidades de 2,500 y 14,999) y rural el $15.30 \%$ (población que vive en localidades menores de 2,500 habitantes). Los municipios con mayor porcentaje de población urbana son; San Mateo Atenco (93.5\%); Toluca (77.28\%) y Metepec (76.51\%), mapa no 1.

\subsection{Características socioeconómicas}

Con base en el Consejo Estatal de Población (COESPO), el grado de marginación y el grado de rezago se registra en forma diferente en la zona de estudio, del total de municipios que conforman la ZMT, nueve tienen muy bajo grado en ambos indicadores, son los que presentan las mejores condiciones sociales; tres municipios registraron bajo grado de marginación y muy bajo grado de rezago, estos son: Calimaya, Xonacatlán y Zinacantepec. Almoloya de Juárez registro grado medio en ambos indicadores, que son condiciones sociales intermedias y Otzolotepec el cual presenta grado medio y bajo respectivamente. Lo que significa que se trata de municipios con necesidades sociales más o menos homogéneas.

De acuerdo al Programa de la Naciones Unidas para el Desarrollo (PNUD), el índice de desarrollo humano, se integra a partir del índice de salud, el índice de 
educación y el índice de ingreso.

Para el 2005 el Estado de México se registró entre las entidades con desarrollo humano alto (IDH mayor o igual a 0.80 ) el cual fue menor al nacional de 0.82 .

Figura 1. Mapa no 1. Distribución de la Población, Zona Metropolitana de Toluca

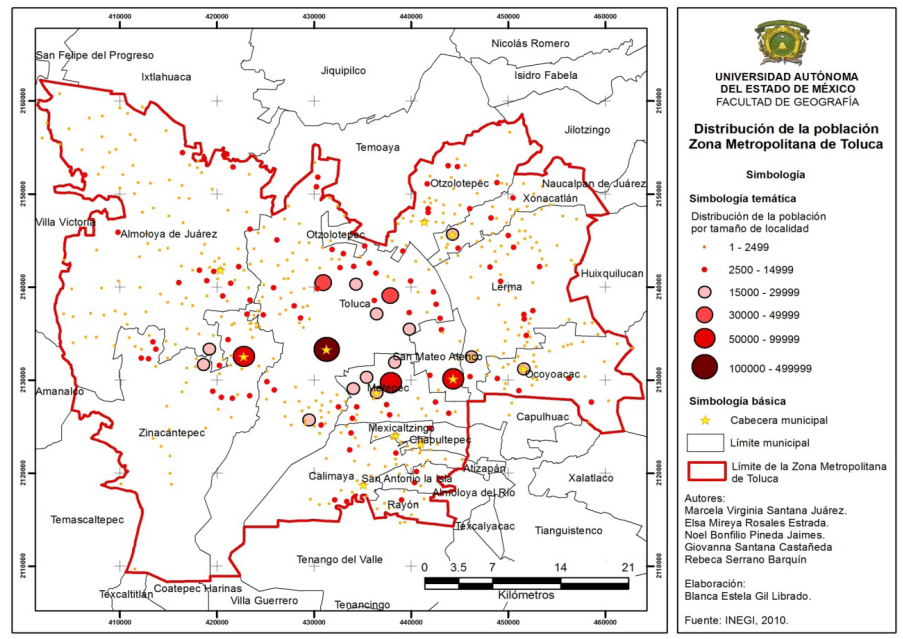

En la ZMT se presentan contrastes, los mejores municipios con IDH muy alto se registraron en Metepec y Toluca; Lerma, Mexicaltzingo, Ocoyoacac, Rayón, San Antonio la Isla, y San Mateo Atenco presentaron IDH alto. Los municipios restantes registraron un IDH medio y bajo, lo que indica que las condiciones sociales son diferenciales en la ZMT.

La globalización tiene efectos en las actividades terciarias, que inciden en el resto de la economía. En este sentido en la zona de estudio se registra un cambio significativo en las actividades económicas de industriales a servicios. Para el 2010, la población ocupada en la ZMT suman 701,638 personas, de las cuales el $64.7 \%$ corresponden al sector servicios; el $31.1 \%$ a industria y el $3.17 \%$ a la agricultura, ganadería, y acuacultura.

En los municipios de Metepec, Mexicaltzingo y Toluca, la población ocupada impera en actividades de servicios, principalmente especializados en educación, comercio y salud, entre otras ramas. En los municipios de Lerma, Otzolotepec y San Mateo Atenco la actividad predominante es la industria. Por su parte los municipios de San Antonio La Isla, Calimaya y Rayón registran una población ocupada 
principalmente en agricultura, ganadería y acuacultura.

\subsection{Características ambientales}

De acuerdo al INEGI (2010), los usos del suelo en esta zona se caracterizan por el predominio de superficie agrícola con el $57.12 \%$ de la superficie total; de pastizal el $10.29 \%$; de bosque el $16.35 \%$; de asentamientos humanos el $16.22 \%$; y de superficie de cuerpos de agua el $0.96 \%$.

Los municipios con los mayores porcentajes de superficie agrícola son: Chapultepec, San Antonio la Isla, Almoloya del Río y Calimaya con más del $77 \%$ de su superficie respectivamente. En relación a la superficie de pastizal el municipio de Mexicaltzingo ocupa el mayor porcentaje de su superficie con el $18.57 \%$. El municipio de Ocoyoacac registra el mayor porcentaje de bosque con el 32.04\% de su superficie. Por otra parte los municipios de San Mateo Atenco y Metepec ocupan los mayores porcentajes del uso de asentamientos humanos con el $43.94 \%$ y el $33.23 \%$ respectivamente.

Por último la superficie de cuerpos de agua que se presentan principalmente en los municipios de Almoloya de Juárez y Toluca que indican el $2.02 \%$ y $1.61 \%$ de sus superficies (mapa no. 2).

Uno de los problemas fuertes actuales en la ZMT es el relacionado con la erosión laminar, de acuerdo a la cartografía de erosión laminar de Juan Castro y Giovanna Santana, (2012), la erosión laminar es fuerte principalmente al oriente y poniente de la ZMT, que coincide con el uso de suelo de bosques de coníferas y al noroeste con uso agrícola y constituyen el potencial para la recarga de mantos acuíferos; por lo que es importante puntualizar estrategias para disminuir la erosión. Los municipios que presentan esta problemática son: Otzolotepec, Xonacatlán, Lerma, Ocoyoacac, Toluca, Zinacantepec y Almoloya de Juárez, (mapa no. 3). 3.4 Salud.

La salud humana como resultado de factores de diversa índole, como los socioeconómicos, ambientales y las políticas públicas que también inciden en ella, y que en este caso se relacionan con la cobertura de salud y los tipos de causas de la mortalidad y morbilidad.

En relación a la cobertura, no se ha incrementado en el IMSS ni en el ISSSTE, en las áreas urbanas; pero se ha incrementado significativamente el seguro popular, principalmente en las áreas rurales. Este último no cuenta con infraestructura 
propia, por lo que se atiende a los pacientes en el IMSS y ello agrava los problemas principalmente de la calidad del servicio en ámbitos urbanos.

Figura 2. Mapa no 2. Usos de suelo de la Zona Metropolitana de Toluca, 2010

\section{Figura: Usos de suelo en la Zona Metropolitana de Toluca, 2010}

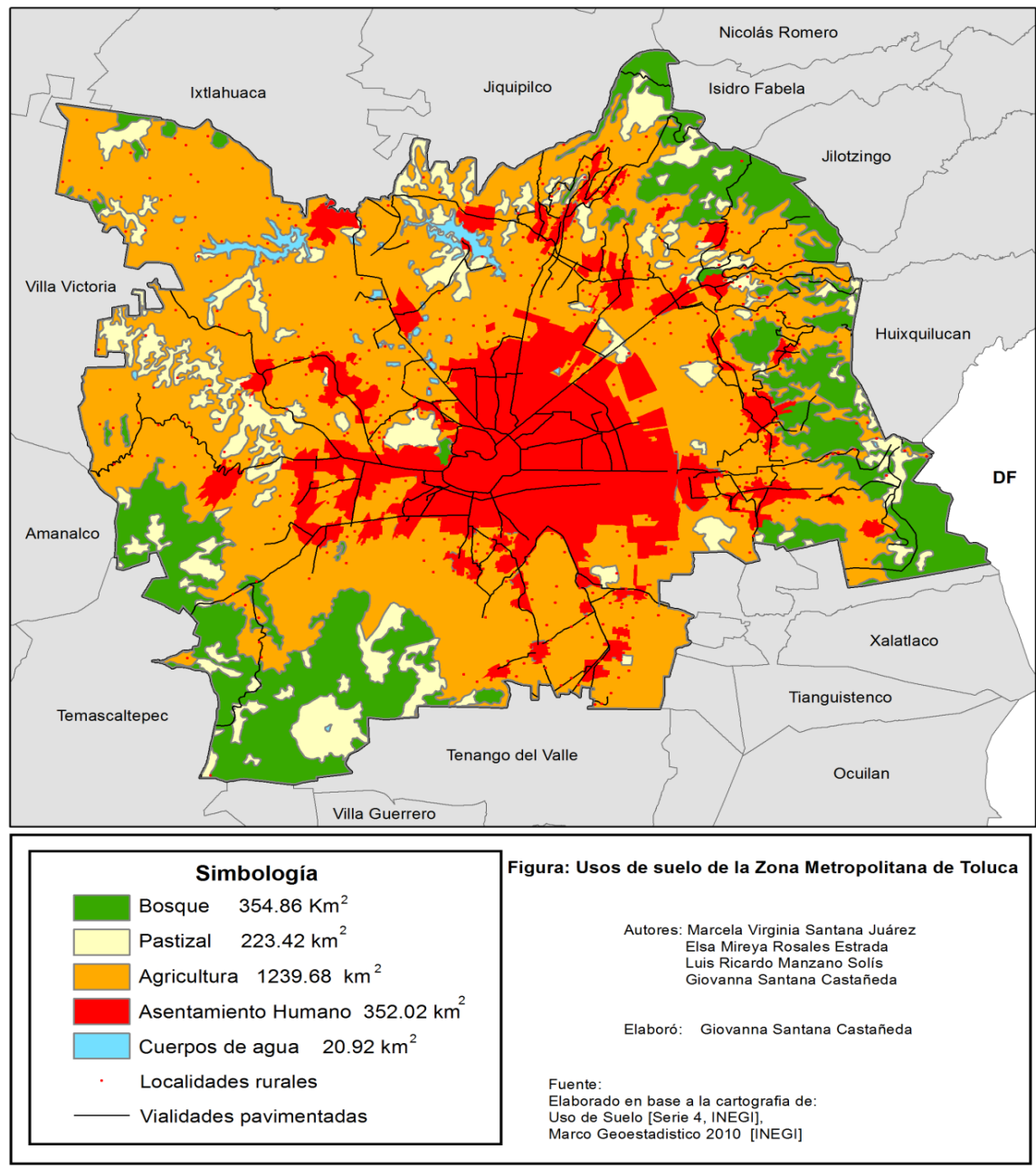


Figura 3. Mapa no 3. Erosión laminar de la Zona Metropolitana de Toluca, 2010
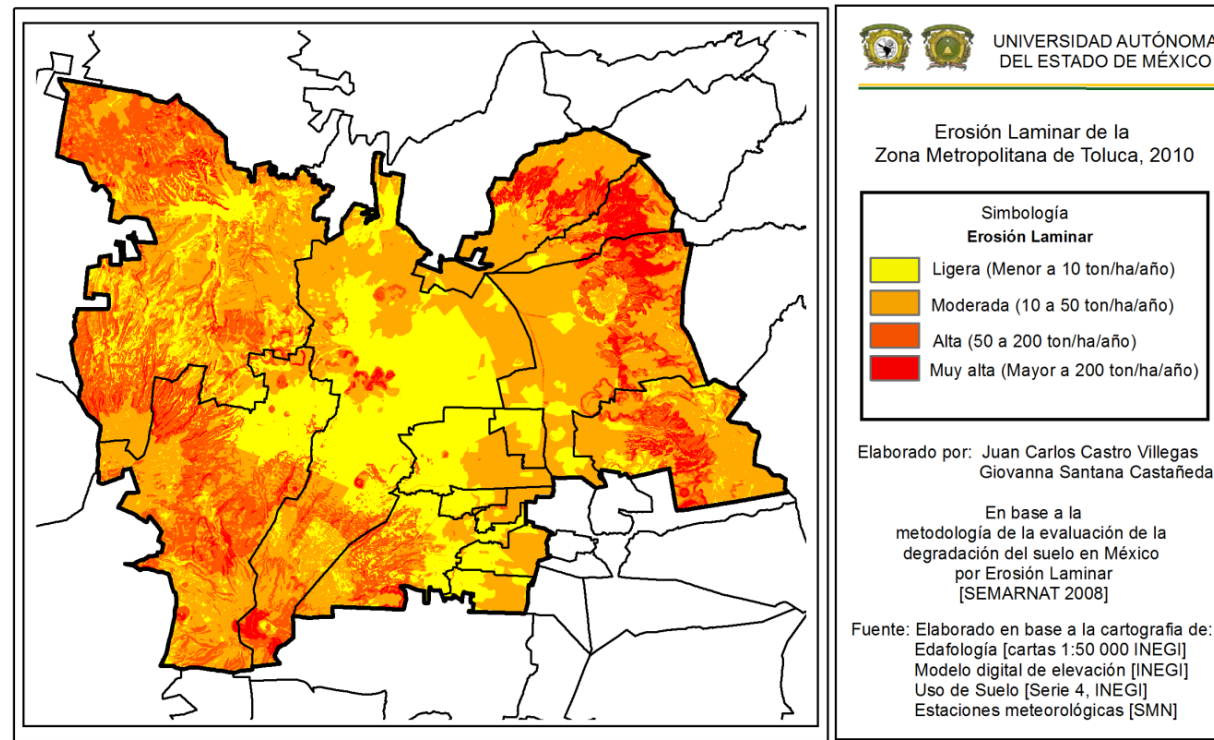

Elaborado por: Juan Carlos Castro Villegas Giovanna Santana Castañeda

En base a la

metodología de la evaluación de la degradación del suelo en México por Erosión Laminar [SEMARNAT 2008]

Fuente: Elaborado en base a la cartografia de: Edafologia [cartas 1:50 000 INEGI]

Modelo digital de elevación [INEGI]

Uso de Suelo [Serie 4, INEG|]

Estaciones meteorológicas [SMN]

Con respecto a la distribución de los servicios de salud, la cobertura a nivel nacional, para el 2010 se incrementó, cubriendo el 64.55\% de la población total. Sin embargo, no se incrementó la cobertura del IMSS, ni del ISSSTE, por lo que el Seguro Popular tiene un papel importante para satisfacer las necesidades de la población principalmente en ámbitos rurales.

A nivel del Estado de México, la población derechohabiente representa el $58 \%$ de la población total: el IMSS cubre el $50.77 \%$ de la población derechohabiente (PDH); el Seguro Popular el 30.15\%; el ISSSTE el 11.61\% y el ISSEMYM el $3.5 \%$.

La distribución de los servicios de salud en la ZMT de Toluca, es cubierta principalmente por el IMSS (45.91\%), por el seguro popular $(32.70 \%)$ y por el ISSSTE (14.59\%) del total de la PDH. La población derechohabiente representa en promedio $64.9 \%$ de la población total. La cobertura por tipo de institución presenta una diferenciación espacial y desigualdades entre los municipios: aquellos con los porcentajes mayores de PDH son Chapultepec (74.69\%), Metepec (68.74\%), San Antonio la Isla $(67.49 \%)$ y Rayón $(66.56 \%)$, en los que predomina la población cubierta principalmente por el IMSS, y en menores porcentajes por el seguro popular. En el caso de Metepec también el ISSSTE tiene participación significativa. 
En el año 2010, la distribución de los recursos humanos para la salud presentó desigualdades significativas. El personal médico en la ZMT suman 3474, que indican el $19.26 \%$ del total estatal. La mayoría se concentra en el municipio de Toluca con 2,601 (el 74.87\% del total de la ZMT). La calidad del servicio se refleja en la proporción de habitantes por médicos. Los municipios de Ocoyoacac, Calimaya y Almoloya de Juárez registran 3090, 2940 y 2171 habitantes por médico en contraste con Toluca que es de $315 \mathrm{hab} / \mathrm{méd}$. El personal médico del IMSS, del DIF, del ISSEMYM y del ISEM se concentra en el municipio de Toluca; del ISSSTE en el municipio de Metepec.

La ZMT incluye 246 unidades médicas que representan el 13.7\% del total estatal. El municipio de Toluca concentra 101 instalaciones y es el $41 \%$ de las unidades médicas de la ZMT, cuenta con 90 de consulta externa, cinco hospitales generales y seis hospitales especializados, (mapa no. 4).

Figura 4. Mapa no 4. Población derechohabiente al servicio de salud
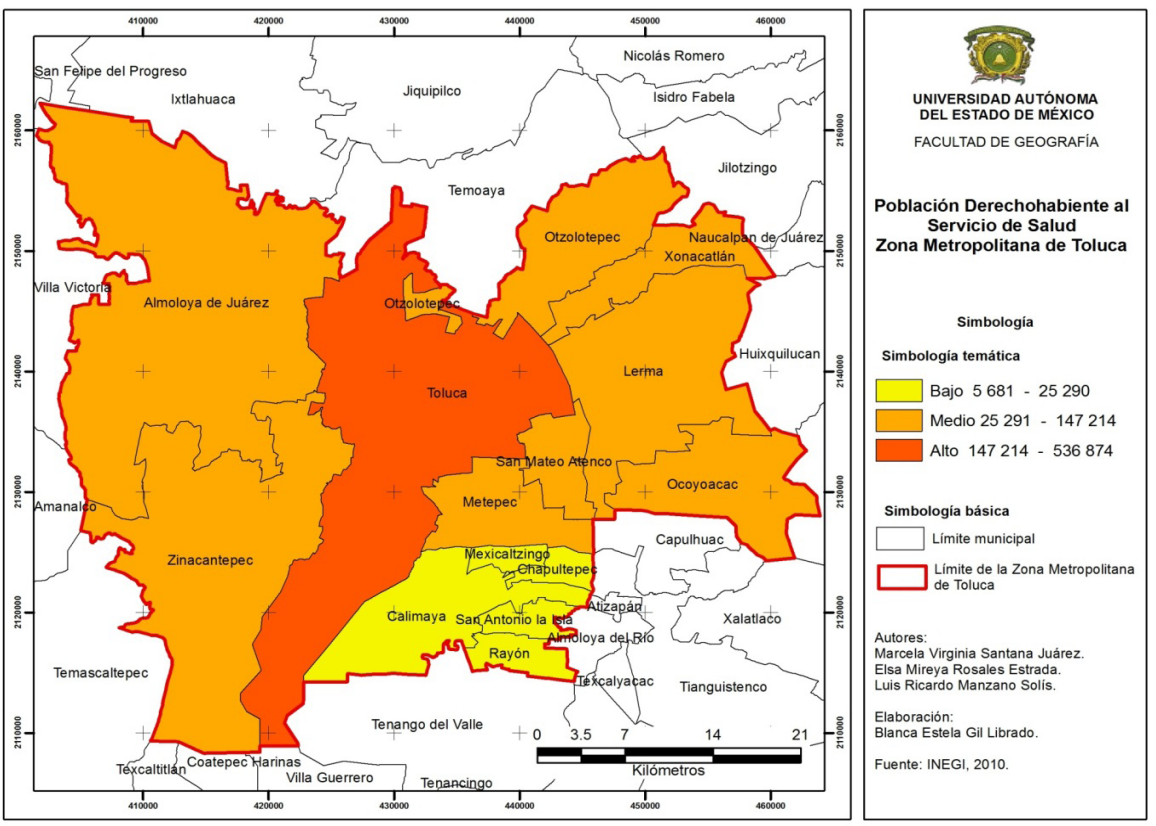

En relación a las causas de mortalidad, estas tienen relación con la obesidad y enfermedades de tipo crónico degenerativo, así como también de tipo infeccioso. 
De acuerdo al Instituto de Salud del Estado de México (ISEM), para el 2010, la ZMT registró un total de 8052 defunciones, con una tasa bruta de mortalidad de 43.61 por 1000 habitantes, inferior a la estatal que fue de 45.0. La distribución de la mortalidad general se presentó en forma diferenciada en el contexto de la ZMT: los municipios que registraron tasa de mortalidad general alta son: Mexicaltzingo (58.91) y Xonacatlán (53.96).

En relación a la mortalidad infantil, se presentaron 663 defunciones de menores de un año, con una tasa de 17 por 1000 nacidos vivos. Los municipios con tasas altas son: Xonacatlán (23), Ocoyoacac (22), Almoloya de Juárez (19), San Mateo Atenco (19) y Zinacantepec (19).

Las causas más frecuentes de mortalidad son de tipo crónico degenerativo: diabetes mellitus, con un total 1552 defunciones y una tasa de 84.7 por 100,000 habitantes, la cual es superior a la estatal; las enfermedades isquémicas del corazón, con 760 defunciones y tasa de 41.17; los Tumores malignos con 577 defunciones y una tasa de 31.25 ; la cirrosis y otras enfermedades del hígado, con 466 defunciones y una tasa de 25.24; por último las enfermedades cerebrovasculares con 362 defunciones y una tasa de mortalidad de 19.61, con tasas inferiores a la estatal.

En relación a la mortalidad específica por grupos de edad, en la ZMT, el grupo más vulnerable es la población pos-productiva, con la mayor tasa de mortalidad, la cual es de 4,955 por 100,000 habitantes; le sigue la mortalidad de menores de un año con 2000 por 100,000; en seguida el grupo de población de 1 a 4 años con 543; posteriormente la población productiva con 261 y por último el grupo de población de 5 a 14 años de edad, con 30.63 por 100,000.

\section{CONCLUSIONES}

En el presente trabajo se enfatizó la salud desde el punto de vista holístico, en la que inciden diversos factores reconociendo al entorno que también incide en el estado físico de la población. Por tanto para conseguir y mantener el bienestar físico, psíquico, social y medioambiental de las personas, se requiere de un entorno natural en equilibrio ecológico, aunado a la infraestructura y personal especializado para la atención de la población, estos factores posibilitan estilos de vida sanos.

La Zona Metropolitana de Toluca con 14 municipios presenta características urbanas y también rurales, sin embargo, se mantienen relaciones de dependencia entre ellos. La ciudad central es Toluca capital del estado más grande en nú- 
mero de habitantes de México. Metepec se presenta con fortalezas desde el punto de vista comercial y de vivienda, Lerma contrasta con un proceso de consolidación industrial, el resto de los municipios compiten estrechamente, cuyas características intrínsecas les proporcionan ventajas relacionadas a los tres municipios mencionados.

De acuerdo a los usos de suelo la mayor parte agrícola y una cantidad pequeña de bosque, circunstancia que evidencia una falta de equilibrio para la captura de carbono en una región de casi dos millones de habitantes.

Cabe destacar que del total de municipios, el 64\% registraron grado de marginación muy bajo; el $85.71 \%$ registró rezago social medio alto y el índice de desarrollo humano, en el $7.1 \%$ es muy alto y el $50 \%$ es alto. Lo que indica que al interior de la ZMT existen diferenciales socioeconómicas.

A pesar de los datos arriba señalados la población con derecho a la asistencia de salud pública en el Estado de México asciende a 58\% y en la Zona Metropolitana es de casi $65 \%$, por lo que todavía aún falta mucho que hacer para mejorar la salud de la población. Se presenta el problema de concentración de recursos, en específico del personal médico, debido a que el $74.87 \%$ se localiza en el municipio de Toluca. Lo mismo sucede con los recursos materiales, como las unidades médicas ya que el $41 \%$ se concentran en este municipio.

En la ZMT, las tasas de mortalidad general son diferenciales, aquellos municipios con las mayores tasas son Mexicaltzingo y Xonacatlán con características socioeconómicas medias y altas. Las tasas específicas de mortalidad también se registran en forma diferencial al interior de la ZMT. En relación a la diabetes mellitus, aquellos municipios con las mayores tasas son Chapultepec, Xonacatlán y Ocoyoacac; por enfermedades isquémicas del corazón las mayores tasas se registran en Toluca, Calimaya y Ocoyoacac. En relación a los tumores malignos las mayores tasas se registran en Mexicaltzingo, Toluca y Metepec con características socioeconómicas altas y muy altas. Con respecto a las enfermedades cerebrovasculares las mayores tasas de mortalidad se registraron en Chapultepec, San Mateo Atenco y San Antonio la Isla. Por último la causa por cirrosis y otras enfermedades del hígado, los municipios con las mayores tasas son Almoloya de Juárez, Chapultepec, San Mateo Atenco y San Antonio la Isla.

La metodología de ordenamiento territorial permite caracterizar esta etapa de la investigación con la finalidad de lograr una visión integral de la salud humana y su especialización en el territorio. A fin de proponer una ordenación territorial para 
la construcción de ciudades saludables que prioricen un crecimiento urbano ordenado y funcional, que incluya espacios públicos, vías de comunicación y transporte funcionales y lugares saludables dignos de las personas que habitamos la ciudad.

Las alternativas de desconcentración de servicios de salud en Toluca se vislumbran complejos, sin embargo la atención especializada en los municipios, de lograr incidir en la atención a cada municipio de acuerdo al perfil epidemiológico se estaría en la condición de transición hacia la construcción de ciudades saludables.

\section{BIBLIOGRAFÍA}

- Castro, Juan y Santana, Giovanna (2012). Cartografía de erosión laminar de los municipios de la Zona Metropolitana de Toluca, 2010. México: Facultad de Geografía, Universidad Autónoma del Estado de México. Inédito.

- Farinós Joaquín (2011). "Capítulo Gobierno: buen gobierno, gobernanza y gobernabilidad de los territorios. Más de lo mismo no será suficiente". En Dinámicas territoriales, políticas de desarrollo territorial sostenible y nueva gobernanza territorial en el espacio Iberoamericano. Conceptos, métodos y tendencias. (Olmos, A; Massiris, A.; Farinós J.; Santana M; Rosales E. Comp.). Toluca, México: II Workshop de la Red Iberoamericana de Observación Territorial, pp. 145-176.

- Gobierno del Estado México (2008), "Seguro popular", [en línea] México. (Consultada el 15 de agosto del 2008). Disponible en: http://www.seguro-popular. salud.gob.mx

- Gobierno del Estado de México (2011). Sistema epidemiológico y estadístico de las defunciones, con base a datos del INEGI/SSA 2010. México: Instituto de Salud del Estado de México.

- Hancock Trevor and Duhl Leonard, (1988). "Promoting health in the urban". WHO healthy cities papers no. Ed. WHO Healthy Cities

- Instituto Nacional de Estadística y Geografía (2010). Censo de población y vivienda 2010. México.

- Instituto Nacional de Estadística y Geografía (2011) Anuario Estadístico del Estado de México. México 
- Massiris, Angel (2011). "El desarrollo territorial sostenible en América Latina: Retos y perspectivas". Dinámicas territoriales, políticas de desarrollo territorial sostenible y nueva gobernanza territorial en el espacio Iberoamericano. Conceptos, métodos y tendencias. (En Olmos, A; Massiris, A.; Farinós J.; Santana M.; Rosales E, comp.). Toluca, México. II Workshop de la Red Iberoamericana de Observación Territorial, pp. 1-8. CD. ISBN: 978-607-00-5058-9, pp. 2-8.

- Organización Mundial de la Salud (1995). Veinte pasos para desarrollar un proyecto de ciudades saludables. Oficina regional de la OMS para Europa.

- Organización de las Naciones Unidas (1992). Agenda 21. Conferencia de las Naciones Unidas sobre el medio ambiente y el desarrollo (UNCED). Río de Janeiro. Brasil.

- Organización Panamericana de la Salud (2005). Municipios, ciudades y comunidades saludables. Recomendaciones para la evaluación dirigida a los responsables de las políticas en las Américas. Washington D.C.: OPS.

- Programa de las Naciones Unidas para el Desarrollo México (2009). "Indicadores de desarrollo humano y género en México 2000-2005". Producción creativa, diseño editorial. Disponible en: http://www.inegi.org.mx/sistemas/productos/ default.aspx?c=265\&s=inegi\&upc=702825042448\&pf=prod\&ef=\&f=2\&cl=0\&t$g=8 \& p g=0>$

- Programa de las Naciones Unidas para el Desarrollo México (2011). "Informe sobre desarrollo humano, Estado de México. Equidad y política social". Disponible en: http://www.undp.org.mx/img/pdf/IDH_Estado de Mexico_2011-2-pdf

- Restrepo, Helena y Málaga, Hernán (2011). Promoción de la salud: cómo construir vida saludable. Ed. American Health Org.

- SEDESOL, CONAPO e INEGI (2008). Delimitación de las zonas metropolitanas de México. México: SEDESOL/CONAPO/INEGI. 